UCRL-JC-125862

PREPRINT

\title{
Compact High-Voltage Structures
}

\author{
M. J. Wilson \\ D. A. Goerz
}

This paper was prepared for submittal to the 11th IEEE International Pulse Power Conference

Baltimore, MD

June 29-July 2, 1997

June 9, 1997

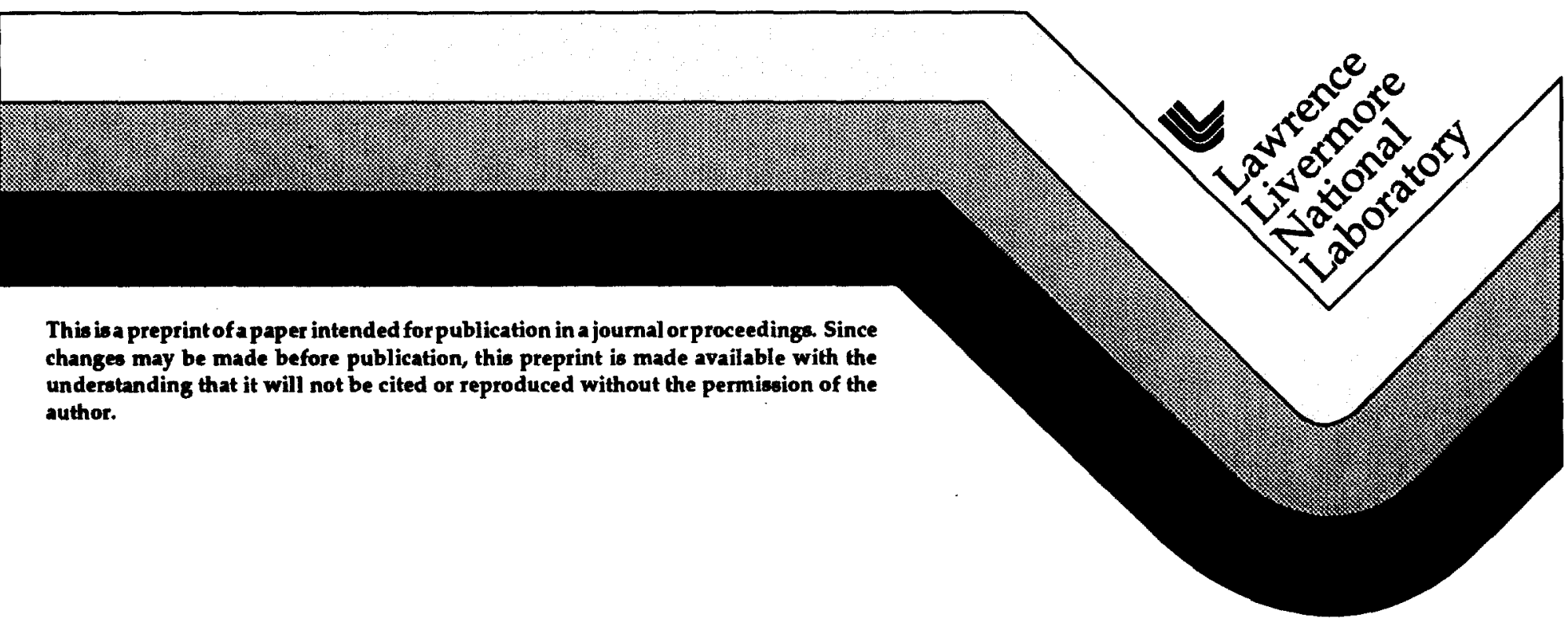




\section{DISCLAIMER}

This document was prepared as an account of work sponsored by an agency of the United States Government. Neither the United States Government nor the University of California nor any of their employees, makes any warranty, express or implied, or assumes any legal liability or responsibility for the accuracy, completeness, or usefulness of any information, apparatus, product, or process disclosed, or represents that its use would not infringe privately owned rights. Reference herein to any specific commercial product, process, or service by trade name, trademark, manufacturer, or otherwise, does not necessarily constitute or imply its endorsement, recommendation, or favoring by the United States Government or the University of California. The views and opinions of authors expressed herein do not necessarily state or reflect those of the United States Government or the University of California, and shall not be used for advertising or product endorsement purposes. 


\title{
COMPACT HIGH-VOLTAGE STRUCTURES ${ }^{*, 1}$
}

\author{
Michael J. Wilson and David A. Goerz \\ Lawrence Livermore National Laboratory \\ P. O. Box 808 (L-153) \\ Livermore, CA $94550-9900$
}

\begin{abstract}
A basic understanding of the critical issues limiting the compactness of highvoltage systems is required for the next generation of impulse generators. In the process of optimizing the design of a highly reliable solid-dielectric over-voltage switch, an understanding of the limiting factors found are shown. Results of a $130 \mathrm{kV}$ operating switch, having a modest field enhancement of $16 \%$ above the average field stress in the switching region, are reported. The resulting high reliability is obtained by reducing the standard deviation of the switch to $6.8 \%$. The total height of the switch is $1-\mathrm{mm}$. The resulting operating parameters are obtained by controlling field distribution across the entire switch package and field shaping the desired point of switch closure. The disclosed field management technique provides an approach to improve other highly stressed components and structures.
\end{abstract}

\section{Introduction}

It is generally accepted that high-voltage devices fail following excessive partial discharge activity ${ }^{2,3}$, which is a localized dielectric breakdown that does not transcend the main electrode gap spacing. The failure is usually anticipated to start at a triple junction, the point at which an electrode and two different dielectric materials intersect. Figure 1 shows a slightly modified view of two different dielectric materials separating two electrodes of different potential. In solid insulation systems the weaker dielectric material can be thought of as an inclusion or nonuniformity in the bulk dielectric. As a voltage is applied between two electrodes a field stress (E) in $V / \mathrm{cm}$ divides across the insulating media as a relationship of

\begin{tabular}{|l|}
\hline upper electrode \\
\hline Solid dielectric \\
\hline lower electrode \\
\hline
\end{tabular}

Figure 1: The above figure illustrates the effect of electrode and insulator interfaces. Typical manufacturing techniques resulting in areas of weaker dielectric material existing between the electrodes and the primary insulation. the capacitance. A simple configuration of a high-voltage structure is an electrode surrounded by air or vacuum, a bulk insulator, air or vacuum, and another electrode at

\footnotetext{
- Work performed under the auspices of the U. S. Department of Energy by the Lawrence Livermore National Laboratory under Contract W-7405-Eng-48.
} 
a different potential. This simple view of a high-voltage structure indicates that a discontinuity exists between the main dielectric and the adjacent electrode. When an applied voltage reaches the dielectric strength of the weaker discontinuous material, partial discharge activity occurs. This voltage level is referred to as the corona inception level or voltage (CIV). Several approaches to mitigate the effects and increase the inception levels are currently used. Most of these concepts include the use of higher dielectric strength materials as impregnates. Oil, pressurized gas (SF6), grease, and encapsulates represent the majority of the materials employed in this process. The basic intent of impregnates is to strengthen the weaker dielectric areas. In contrast removing these areas provide structural improvement by eliminating or excluding the field between the electrode and the bulk dielectric material.

\section{Approach}

A recent experimental effort involving an over-voltage solid-dielectric switch demonstrated increased failures as operating levels approached the design goals. A failure rate of $30 \%$ indicated that the selected material, polyethylene terephthalate (mylar), had an estimated standard deviations $(\sigma)$ of 18 to $20 \%$. A random inspection of the mylar shows that the available lot in a controlled test fixture approaches $\sigma$ level of $8 \%$. Previous work ${ }^{4,5}$ also indicates that in a controlled configuration mylar shows a $\sigma$ of 8 to $12 \%$. The authors found it interesting that after fifty years of manufacturing experience, a significant improvement in the breakdown distribution of mylar film had not been realized. A circuit simulation indicated that the optimum $\sigma$ for a reasonable reliable system needed to be 4 to $6 \%$. This level of control provides a $99 \%$ probability of success with acceptable circuit variables.

In the process of preparing to test breakdown levels of a dielectric material between two planar electrodes, we performed measurements of the capacitance of the specimen and found a pronounced difference between measured and calculated values. Further consideration revealed that an estimated air gap of $12.7-\mu \mathrm{m}(0.0005$ inches) would account for the difference in the observed capacitance. The flatness of the electrode was measured which indicated that this could easily be the cause for the difference. As indicated in Figure 1, the existence of an air gap between the electrode and principal dielectric will result in a concentration of fields in the lower dielectric constant medium. Before the intrinsic breakdown level of the solid dielectric can be reached, the lower dielectric strength gas in the gap will breakdown. Ideally, one would like to completely eliminate all anomalies between the dielectric-electrode interface, but usual manufacturing methods do not accomplish this. The solution we implemented was to metalize both surfaces of the dielectric material before sandwiching it between the electrodes. This eliminates the electric field in any gap that may be present.

The issue then becomes what are the contributors to the switching parameters and can they be engineered to the desired level. Using 3 as a typical value for the dielectric constant, a minimum thickness for this specific application was found to be $1-\mathrm{mm}$. Typical mylar film is available in thickness up to $0.5-\mathrm{mm}$ (0.020 inches). The concern of 
inclusions as illustrated in Figure 1 between multi-layer films eliminated mylar as a possible design candidate. As a result other-dielectric materials were investigated. The desired nominal operating voltage is $100 \mathrm{kV}$. Typical solid-dielectric materials easily handle these levels of stress. The design would need to include a controlled region for breakdown. Three materials were selected for evaluation, polycarbonate, acrylonitrile/butadiene/styrene resin (ABS) mixes ABS-4410, and ABS-9450P.

\section{Test/Results}

The main emphasis for this report is to show the improved operating characteristics of the selected switch configuration. Several different types of testing were used to assess the level of controls needed in the manufacturing of the final design. The testing included: visual and mechanical inspection, $x$-rays, partial discharge analysis, and breakdown measurements.

The following table summarizes the test results for the four selected dielectric candidates. A typical testing protocol for candidate evaluation is to prepare 100 switches, visually and mechanically inspect, x-ray, prepare and metalize surfaces, reinspect, measure lot samples for partial discharge activity, clean, impulse stress to breakdown, and analyze data.

Table 1: The following table provides a summary of the test results of possible soliddielectric switch candidates. The measured levels are average values obtained after an optimizing screening process were applied to the investigated material. The field stress level takes into account the enhancement factor of the switching region.

\begin{tabular}{|c|c|c|c|c|}
\hline Material & $\begin{array}{c}\text { Measured } \\
\text { voltage } \\
\text { breakdown } \\
\text { level }(\mathrm{kV})\end{array}$ & $\begin{array}{c}\text { Resulting } \\
\text { maximum field } \\
\text { strength } \\
(\mathrm{MV} / \mathrm{cm})\end{array}$ & $\begin{array}{c}\text { Breakdown } \\
\text { voltage } \\
\text { standard } \\
\text { deviation }(\%)\end{array}$ & $\begin{array}{c}\text { Measured } \\
\text { dimple profile } \\
\text { standard } \\
\text { deviation }(\%)\end{array}$ \\
\hline Polycarbonate & 114 & 5.23 & 14.87 & 2.65 \\
\hline ABS-4410 wet & 69 & 3.19 & 10.80 & 2.23 \\
\hline ABS-4410 dry & 109 & 5.02 & 8.50 & 2.23 \\
\hline ABS-9450P dry & 164 & 7.54 & 6.81 & 2.08 \\
\hline
\end{tabular}

\section{Polycarbonate}

Polycarbonate represents a versatile material used for its machining and electrical characteristics. This was the first material selected for this application. The tolerances of the dimple region and the plastic rebound of the material resulted in a widening of the statistical distribution of the breakdown profile. A mechanical inspection technique typically used to profile surfaces showed that the dimple region at the base was flat and not contoured as designed. It is believed that the resulting profile added significantly to the operational $\sigma$ of $14.9 \%$. The explanation for this effect is that in a blind hole or bottom machining process the cutting tool does not cut the material in the line of axial machining. The resulting part to part repeatability for this manufacturing technique was 
measured to have a $\sigma$ of $2.65 \%$. In addition slightly higher cost was required to put down the conductive surfaces. This configuration was not considered to be an attractive solution.

\section{ABS-4410 wet (acrylonitrile/butadiene/styrene resin)}

ABS-4410 represents one of the most widely used injection mold plastics. The initial run was not dried during the process. The results are given in summary as a comparison. The same mechanical inspection profile measurement was performed on a representative sample of 100 test specimens. The resulting $\sigma$ for this measurement of the dimple region is $2.23 \%$. The measured dimple contour shows the desired design profile and overcame the machining problems of polycarbonate. This level of repeatability and ability to contour complex shapes show the attraction to this manufacturing process. The operational level however did not meet the design requirements and a plating grade $A B S$ was procured for evaluation.

\section{$A B S-4410 d r y$}

A second run of dried ABS-4410 was made to measure the added value of drying during the manufacturing process. The breakdown voltage $\sigma$ measured on 100 screened switches is $8.5 \%$. The added process of drying the plastic before injection improved the operational characteristics to a level of possible consideration.

\section{$A B S-9450 P$ dry}

ABS-9450P is the plating grade type of injection mold plastic that was evaluated. The preferred method of assessing manufacturing techniques is to request standard manufacturing controls. The thought is that added controls will increase the cost and may not be needed. The surface profile and dimple region were measured with the established technique. The resulting $\sigma$ for the dimple region is measured to be $2.09 \%$. The breakdown measurements indicated an impressive field strength, operating level, and the desired reliability having a $\sigma$ of $6.8 \%$ for the breakdown voltage.

The use of partial discharge measurement activity (PDA) is included as part of a possible screening process. Figure 2 provides a graphical view of the observed decrease in $\sigma$ as a decrease in ratio of breakdown voltage to peak corona

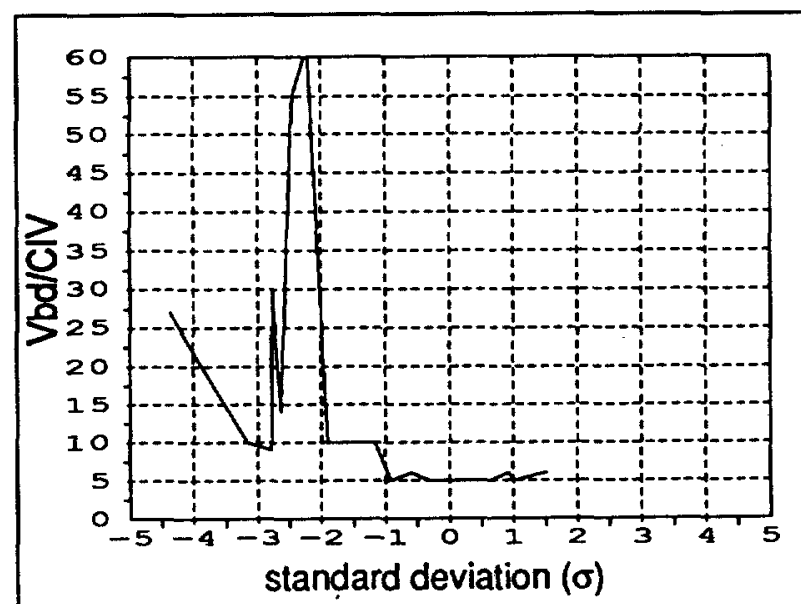

Figure 2: The above illustration shows the added view of using partial discharge measurement to establish a screening level to improve reliability. The vertical scale is given by the ratio of $\mathrm{Vbd} / \mathrm{CIV}$ and the horizontal scale is in standard deviation from the average operating level. 
inception value ( $\mathrm{Vbd} / \mathrm{CIV}$ ). The conclusion from this data indicates that it is possible to screen out the lower population and truncate the distribution. The level of improvement appears to reduce the minimum operating level to a single value of $\sigma$. A complete view of the data indicates that this screening process initially be applied to the entire population. This is required to catch the drop out units not screened by other inspection criteria.

The principal parameter of interest for this work deals specifically with the level of control in the material breakdown. Figure 3 shows the measured cumulative distribution sigmoid of the entire population of ABS-9450P switches. Explaining the observed step in the distribution illustrated in Figure 3 indicates that the units may have undergone a production change during the plating process. In the review of the plating process it was noted that a double process of the first lot of switches occurred. A clearer view of the indication is obtained from a serial view of the population as indicated in Figure 4. The selected processing involved three lots of switches. After the first lot was processed an independent attempt to improve the lot by replating was undertaken. This involved etching the ABS again for metalization. As a result two distinctive breaks in the data are observed, one at $130 \mathrm{kV}$ and the other at $165 \mathrm{kV}$. The double process units

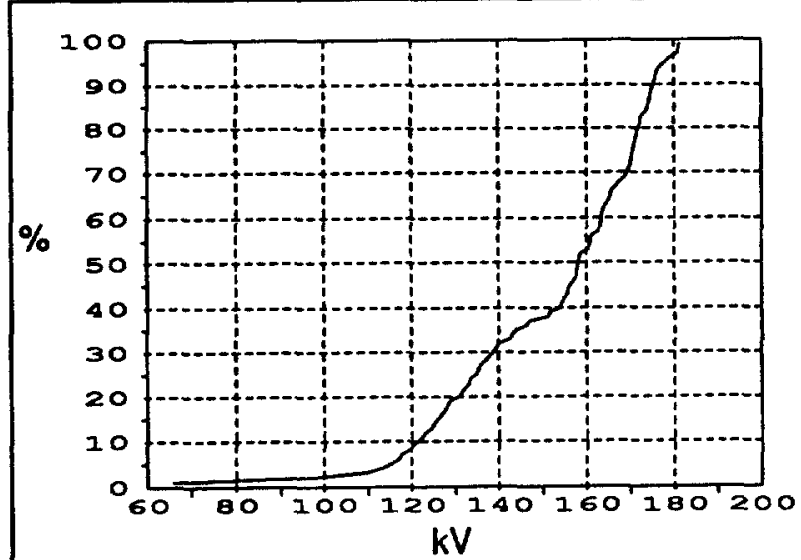

Figure 3: The above cumulative distribution represents the entire population of metalized soliddielectric switches that were tested before screening values are applied. The vertical scale is in percentage of population and the horizontal scale is breakdown voltage in $\mathrm{kV}$.

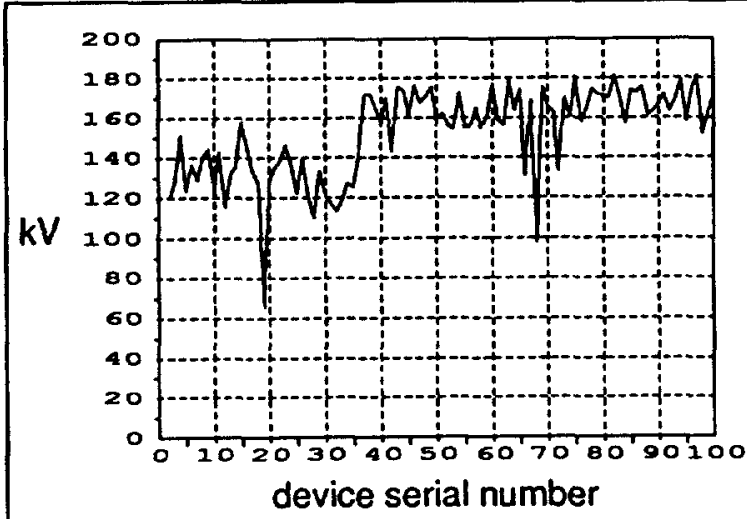

Figure 4: The above figure connects the peak levels of the histogram of the entire population of ABS-9450P switches. The vertical scales showing the breakdown voltage is given in $\mathrm{kV}$ and the horizontal scale are serial processed switches by serial number.

were screened out for the final analysis. Two drop outs (serial numbers 19 and 68) were not screened out of the population by visual inspection or other means and as a result a PDA or an alternative high-voltage screening appears required. 


\section{Summary Materials}

A summary of all the above switches built to the same design and specified tolerances are shown in Figure 5. The vertical scale is given as a relative height (n) of a normal distribution relating to the projected value of a screened distribution. The horizontal scale is in $\mathrm{kV}$. The four test candidates are normalized and placed on the same horizontal axis for direct comparison. The quality and value of each candidate are shown by the distribution and the resulting operating range. The operating parameters of each candidate become obvious and the selections of the manufacturing process and material is now possible. A design iteration is required to fabricate proper operating parameter device. This involves adjusting the height of the dimple region from the opposing electrode for the desired operating level.

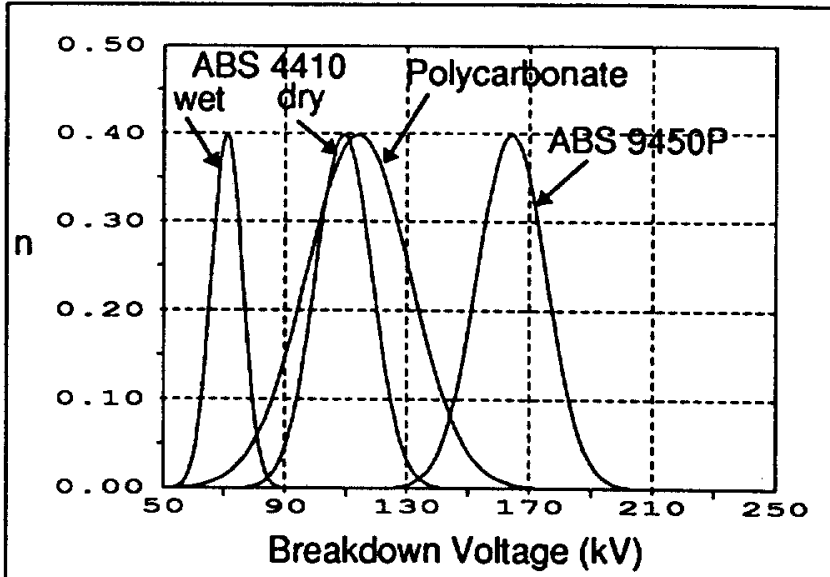

Figure 5: The above figure illustrates a comparison of the measured switch voltage breakdown. The vertical scale $(n)$ is the relative height of a normal distribution relating to the standard deviation. The horizontal scale is in $\mathrm{kV}$ and indicates the level of breakdown.

\section{Conclusion}

It has been shown that metalizing the surface of a solid dielectric that would otherwise be adjacent to an electrode eliminates the fields that would exist in the gap between the dielectric and electrode, thus avoiding breakdown in the weaker (gaseous) dielectric region. It has also been demonstrated that an increase in component reliability results from this removal increasing performance. Manufacturing controls play a critical role in production and a variance from the established process may adversely effect the performance. Visual screening techniques are often valuable but not sufficient for identifying all substandard components. This example uses standard printed circuit board plating and etching techniques. The use of available manufacturing techniques provides solutions to previously accepted effects and limits. The result of improved field management provides an increase in reliability and compactness of high-voltage components and structures.

\footnotetext{
'This report contains information included in pending patents: Wilson, M. J. and D. A. Goerz (1997), "Compact High-Voltage Structures," 11th IEEE International Pulsed Power Conference, Baltimore, MD, Lawrence Livermore National Laboratory, Livermore, CA, UCRL-JC-125862.

${ }^{2}$ L. L. Alston, High-Voltage Technology (Oxford University Press, London, Great Britain, 1968).

${ }^{3}$ R. Bartnikas, E. J. McMahon, Engineering Dielectrics: Vol. I, Corona Measurement and Interpretation (American Society for Testing and Materials (ASTM) STP 669, 1979).

J. C. Martin, "Notes on Some Solid Dielectric Switches," S. S. W. A. AERE Aldermaston, England, May 31, 1962.

5. C. Martin, "Solid Dielectric Pulse Breakdown," Hull Lecture Notes, Hull University, June, 1973.
} 


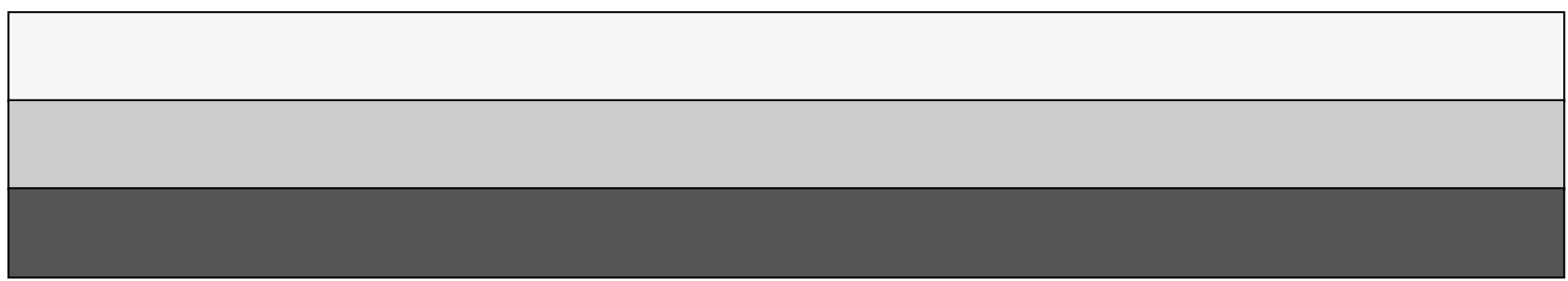

